\title{
A comparative analysis of single port versus multi-port robotic assisted radical prostatectomy for prostate cancer
}

The surgical management of prostate cancer $(\mathrm{PCa})$ has seen a seismic shift over the past number of decades. From the development of the perineal prostatectomy at the start of the last century and its adoption for PCa treatment, to Walsh's modification of the retropubic transperitoneal approach in the 1970's, to the wide scale adoption of the robotic platform at the turn of this century. Despite the lack of evidence to support improved functional or oncological outcomes with the robotic approach over $80 \%$ of prostatectomies in the United Kingdom are now performed robotically (BAUS.org.uk). The benefits of this minimally invasive approach are predominantly linked to the smaller incisions reducing pain and length of hospital stay.

With regards to cancer survivorship the patient does not bear the same psychological and physical scars with minimally invasive surgery (MIS) compared to open surgery. MIS techniques are constantly advancing and we are seeing a resurgence in natural orifice transluminal endoscopic surgery and a push to single port (SP) robotic platforms. Initial attempts with SP surgery were hampered by external clashes, restricted access for the assistant and poor ergonomics with difficulty around intracorporeal triangulation of instruments. With the advent of robotics and its addition to SP surgery many of these challenges are being resolved.

A non-systematic review of literature utilising the search terms 'single port' OR 'single site' AND 'prostate' AND 'robot' returned 90 studies, a third of which pertain to radical prostatectomy. Conversely using the search terms 'robotic' AND 'prostate' AND 'Radical' yields almost 1,800 studies. To date there has not been wide-scale adoption of the SP technique and this is reflected in the literature. Two-thirds of studies are single case reports or case series and feasibility studies with only seven studies containing 20 or more subjects. Four studies utilise a cadaveric model in the early exploratory phase of novel prostatectomy techniques with the addition of the robotics and SP surgery. These include the addition of the SP robot to standard perineal prostatectomy and transperitoneal SP retzius sparing prostatectomy [1,2]. Eight reviews on SP radical prostatectomy exist with one being a systematic review. In this systematic review only six papers met the inclusion criteria. One comparative study explores outcomes of extra- and trans-peritoneal SP radical prostatectomy. Six studies solely focus on perineal prostatectomy and discuss the addition of the SP platform; all are small feasibility studies or reviews. There are no studies comparing standard and robotic perineal prostatectomy outcomes.

To date only two retrospective single institution studies directly compare SP and multi-port (MP) robotic radical prostatectomy outcomes. Vigneswaran et al. [3] found significantly less pain on post-operative day one and shorter hospital stay (1 vs. 2 days) in the SP group with equivocal operating time, positive surgical margin (PSM) and complication rates. Five SP cases were performed via an extraperitoneal approach. While lymph node yields were similar between groups significantly more patients had a lymph node dissection on the MP platform (50\% vs. $75 \%$ ). Functional outcomes at 90 days was measured in term of pad usage and no difference existed between the SP and MP platforms.

Saidian et al. [4] focused their study on the primary outcome of procedural safety and complications and secondary outcomes of operative times, estimated blood loss, PSM rate, functional and oncological outcomes at 90 days. They again published no significant difference in post-operative erectile function or continence outcomes between the two platforms at 90 days. They also examined PSA nadir at 90 days and while not statistically significant found detectable PSA in $7.5 \%$ of the SP cohort vs. $2.2 \%$ of the MP cohort. This is likely related to the higher rate of extraprostatic extension and higher proportion of T3 found in the SP cohort although PSM were equivocal across both groups. Despite 
this only $68 \%$ of the SP cohort underwent pelvic lymph node dissection (PLND) compared to 92\% of the MP group. While complication rates were identical in both groups, there was a higher incidence of lymphocele formation in the SP cohort (15.5\% vs. $4.5 \%$ of PLND in SP and MP cohort respectively).

Both studies are limited by their small sample size making meaningful statistical analysis challenging and also selection bias due to the non-randomised nature of the retrospective studies. The final comparative study compares the extra- and trans- peritoneal approaches in SP robotic radical prostatectomy. They found a significantly shorter operative time, shorter hospital stay ( 4.3 vs. 25.7 hours $p<0.0001$ ) and less opioid based pain requirements with the extraperitoneal approach. It is postulated that the dramatically shorter hospital stay and reduced pain requirements in the extraperitoneal cohort is due to the reduced peritoneal irritation and gastrointestinal side effects as compared the transperitoneal approach also requiring steep Trendelenburg positioning. This was similarly seen in studies comparing extra- and trans-peritoneal MP robotic prostatectomy. PSA levels and continence outcomes at 90 days showed no statistical difference between the extra- and intra- peritoneal prostatectomy groups [5].

The sole systematic review and pooled analysis on SP radical prostatectomy included six studies in the final analysis and excluded all robotic perineal surgery [6]. The number of cases in the series examined ranged from 3-49 making meaningful assessment of complication rates, PSM rate and functional outcomes difficult. Mean operative times, estimated blood loss, hospital stay and length of catheterisation were in keeping with large MP radical prostatectomy series [7] as was PSM rate. One study reviewed included a solitary salvage prostatectomy with no conversion to open [4].

To date studies investigating SP robotic radical prostatectomy lack sufficient numbers to perform meaningful statistical analysis but preliminary data suggests that it is a safe procedure with comparable oncological and functional outcomes to standard MP surgery. There is no compelling data to establish its superiority over the MP technique, however there is also no substantial data to support the superiority of any form of robotic radical prostatectomy over the open retropubic technique, yet the procedure has superseded its open counterpart dramatically in the western world.

The new Da Vinci SP robotic system overcomes many of the initial problems surrounding SP surgery such as external instrument clashes, reduced dexterity and precision and loss of intracorporeal triangulation. The learning curve for SP surgery in established MP surgeons appears short and the benefits to the patient of reduced scarring, less pain and shorter hospital stay make this approach very attractive. Future larger scale studies are needed to establish further benefits to the standard MP approach.

\section{CONFLICTS OF INTEREST}

The authors have nothing to disclose.

\section{AUTHORS' CONTRIBUTIONS}

This work was written by Anna L Walsh in collaboration with Prokar Dasgupta who oversaw the contents and editing of the body of work.

Anna L Walsh ${ }^{1}$ (i), Prokar Dasgupta ${ }^{2}$ (i) Corresponding Author: Anna L Walsh ORCID: https://orcid.org/0000-0002-9239-5913

${ }^{1}$ Department of Urology, Guy's and St Thomas' NHS Foundation Trust, London, ${ }^{2}$ Faculty of Life Sciences and Medicine, King's College London, London, United Kingdom E-mail: anna.walsh@gstt.nhs.uk

\section{REFERENCES}

1. Kaouk JH, Akca O, Zargar H, Caputo P, Ramirez D, Andrade H, et al. Descriptive technique and initial results for robotic radical perineal prostatectomy. Urology 2016;94:129-38.

2. Ng CF, Chan ESY, Teoh JYC. The use of the da Vinci SP system for retzius-sparing radical prostatectomy in cadaveric model. Urology 2019;125:260.

3. Vigneswaran HT, Schwarzman LS, Francavilla S, Abern MR, Crivellaro S. A comparison of perioperative outcomes between single-port and multiport robot-assisted laparoscopic prostatectomy. Eur Urol 2020;77:671-4.

4. Saidian A, Fang AM, Hakim O, Magi-Galluzzi C, Nix JW, RaisBahrami S. Perioperative outcomes between single-port and multi-port robotic assisted radical prostatectomy: a single institutional experience. J Urol 2020 Feb 24 [Epub]. https://doi. org/10.1097/JU.0000000000000811.

5. Kaouk J, Aminsharifi A, Wilson CA, Sawczyn G, Garisto J, Francavilla $S$, et al. Extraperitoneal versus transperitoneal single port robotic radical prostatectomy: a comparative analysis of perioperative outcomes. J Urol 2020;203:1135-40.

6. Checcucci E, De Cillis S, Pecoraro A, Peretti D, Volpi G, Amparore $D$, et al. Single-port robot assisted radical prostatectomy (SP-RARP): a systematic review and pooled analysis of the preliminary experiences. BJU Int 2020 Apr 4 [Epub]. https://doi. 


\section{ICUROLOGY}

org/10.1111/bju.15069.

7. Tholomier C, Couture F, Ajib K, Preisser F, Bondarenko HD, Negrean C, et al. Oncological and functional outcomes of a

\section{Single vs. multi-port robotic radical prostatectomy}

large Canadian robotic-assisted radical prostatectomy database with 10 years of surgical experience. Can J Urol 2019;26:984351. 